\title{
BIFOCAL PACING - A NOVEL CARDIAC RESYNCHRONIZATION THERAPY? RESULTS OF BIFOCAL PACING STUDY AND REVIEW OF THE CURRENT LITERATURE
}

\author{
Alan Bulava, Jan Lukl

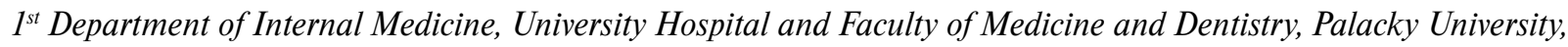 \\ Olomouc, Czech Republic \\ e-mail:alanbulava@seznam.cz
}

Received: April 28, 2006; Accepted: September 9, 2006

Key words: Cardiac resynchronization/Bifocal pacing/Biventricular pacing/Hemodynamics

Background: Bifocal pacing (BFP) has been proposed as a more feasible alternative of cardiac resynchronization therapy (CRT).

Aim: To evaluate BFP in patients with severe congestive heart failure and significant intraventricular conduction delay and to compare it with biventricular pacing (BVP).

Methods: Both echocardiographic examination including tissue Doppler imaging and invasive measurements of cardiac hemodynamics was performed under basal conditions and during BFP and BVP.

Results: 50 patients were included: 29 patients with ischemic heart disease (IHD), 21 patients with idiopathic dilated cardiomyopathy (IDCM). Left ventricular (LV) pressure gradient $\left(\mathrm{dp} / \mathrm{dt}_{\max }\right)$ increased during BFP compared to the baseline $(13.4 \%, 95 \%$ CI 9.2-17.6\%, p < 0.0001) and a further increase was achieved during BVP $(29.5 \%, 95 \%$ CI $23.7-35.4 \%, \mathrm{p}<0.0001)$. A significant correlation was found between the distance of the right ventricular apical and outflow tract leads and percentage of $\mathrm{dp} / \mathrm{dt}$ increase in IDCM patients $(\mathrm{r}=0.72, \mathrm{p}<0.001)$, but not in IHD patients. Interventricular mechanical delay (IVMD) decreased in BFP compared to baseline (43 $\pm 22 \mathrm{~ms}$ vs. $53 \pm 31$ $\mathrm{ms}, \mathrm{p}=0.006)$. BVP produced even shorter IVMD $(22 \pm 19 \mathrm{~ms}, \mathrm{p}<0.0001)$. In all patients, the regional systolic contraction times were significantly shortened, corresponding with prolongation of the respective regional diastolic filling times during both BFP ( $p<0.05$ for all segments) and BVP ( $<<0.001$ for all segments). The effect of BVP on regional systole shortening was more pronounced.

Conclusions: BFP improves LV hemodynamics by decreasing the inter- and intraventricular conduction delays. The leads in the right ventricle should be placed at the longest achievable distance. BVP is superior to BFP.

\section{INTRODUCTION}

Biventricular pacing (BVP) has been an established form of resynchronization therapy in patients with advanced congestive heart failure and intraventricular conduction delay ${ }^{1}$. However, the procedure is reported to be technically unsuccessful in up to $5-13 \%$ of cases $^{2-4}$. Similarly, bifocal pacing (BFP), i.e. simultaneous AV synchronous pacing of the right ventricular apex and the right ventricular outflow tract, has been recently proposed as a possible resynchronization alternative in such patients ${ }^{5,6}$. The aim of this study was to evaluate bifocal pacing as a modality of cardiac resynchronization therapy in patients with severe congestive heart failure and significant intraventricular conduction delay and to compare its benefits with biventricular pacing.

\section{METHODS}

The local ethical committee approved the study. Patients indicated for cardiac resynchronization therapy according to entry criteria (Tab. 1) were included in the study. After signing written informed consent they underwent implantation of temporary bifocal pacing leads. Two quadrupolar electrophysiological catheters were introduced via right femoral vein under local anesthesia and were carefully positioned in the right ventricular apex (RVA) and in the right atrial appendage (RAA). Another active-fixation lead (Pacesetter 1488T $58 \mathrm{~cm}$ ) was introduced via the right internal jugular vein and fixed in the high right ventricular outflow tract (RVOT). The target position was defined fluoroscopically: The lead was introduced under fluoroscopic guidance in the anteroposterior projection above the pulmonary valve. Afterwards, it was withdrawn just beneath the pulmonary valve with a counter clockwise rotation. Such a maneuver was controlled in both anteroposterior (Fig. 1A) and left anterior oblique views (Fig. 1B) to be sure that the lead was oriented and screwed-in the high interventricular septum, not into anterior or free right ventricular wall. Good pacing and sensing parameters and stability had to be also achieved. After echocardiographic acquisition was performed (see beneath), patients underwent implantation of a biventricu- 
lar pacemaker. The mid-lateral or posterolateral branch of the coronary sinus was selected as a target vein for implantation of the left ventricular (LV) lead in all cases.

Hemodynamic measurements during bifocal and biventricular pacing were conducted using a pigtail catheter introduced via the left femoral artery into the left ventricle and aorta and connected to a pressure transducer. The catheter was flushed with saline infusion with heparin to ensure its patency. The maximum rate of LV pressure rise $\left(\mathrm{dp} / \mathrm{dt}_{\text {max }}\right)$ and aortic pulse pressure (Ao-PP) were measured during basal state, during right ventricular apical pacing (RVAP), right ventricular bifocal pacing (BFP) and biventricular pacing (BVP). All ventricular pacing was performed in VAT mode, i.e. atrial sensed AV synchronous stimulation at the intrinsic heart rate with $\mathrm{AV}$ delay set to $120 \mathrm{~ms}$ to ensure full preexcitation of the ventricles. The order of hemodynamic measurements in different stimulation modes was randomly selected using a simple software routine. A steady state of five minutes was required before data were acquired in each modality. The values were measured three times and than averaged. Variability of measurements was calculated in each pacing modality and during basal state. Care was taken to exclude all premature cardiac beats from the measurement. As tipmanometer catheters were not used in this study, percentage of $\mathrm{dp} / \mathrm{dt}_{\text {max }}$ increase was calculated during bifocal and biventricular pacing modalities with respect to the basal measurements and the percentage, not absolute values, were afterwards used for statistical analysis.

QRS complex duration was evaluated on the surface 12-lead electrocardiogram (ECG) recorded during bifocal and biventricular pacing (AV delay $120 \mathrm{~ms}$ ) as well as during intrinsic rhythm. The longest QRS complex in the precordial leads was used for QRS duration measurement.

\section{Echocardiographic measurements}

Standard two-dimensional (2-D), Doppler and tissue Doppler imaging (TDI) echocardiography were performed according to the guidelines of the American Society of Echocardiography ${ }^{7,8}$ with a commercially available ultrasonographic system (Vingmed 7, GE Medical). All three modalities of left ventricular activation (i.e. intrinsic rhythm, bifocal pacing and biventricular pacing) were compared. Left ventricular ejection fraction (LVEF) was measured using the Simpson rule from apical 4- and 2-chamber views. Detailed echocardiographic recordings were obtained for off-line measurement of the following parameters on traditional pulsed wave (PW) flow Doppler recordings: the global $\mathrm{CO}$ interval (i.e. the interval lasting from the mitral valve closure to its reopening, which was measured from the end of the A-wave of the preceding cycle to the beginning of the E-wave of the subsequent cycle); the EA interval (i.e. the interval from the beginning of the E-wave to the end of the A-wave); the Q-A interval (i.e. the interval from the Q-wave on the surface electrocardiogram to the end of the aortic flow); the LVET (i.e. the left ventricular ejection time, measured as the duration of the aortic flow); the LPEP (i.e. the left ventricular pre-ejection time measured from the $\mathrm{Q}$-wave to the beginning of the aortic flow). The Q-P, RVET (right ventricular ejection time) and RPEP (right ventricular pre-ejection time) intervals were measured in a similar manner on pulmonary PW Doppler recordings. Aortic $\left(\mathrm{VTI}_{\mathrm{AO}}\right)$ and pulmonary $\left(\mathrm{VTI}_{\mathrm{PU}}\right)$ velocity time integrals were also calculated in each pacing modality and in intrinsic rhythm. Myocardial performance index was calculated according to Tei' .

The TDI method used in our study for evaluating resynchronization benefits has been described in detail elsewhere ${ }^{10}$. Briefly, TDI analysis was performed in curved M-mode color and PW modalities. Five basal segments of the left ventricle (septal [IVS], inferior [INF], posterior [POST], lateral [LAT], and anterior [ANT]) were evaluated with respect to the regional events. We measured: - the regional $\mathrm{CO}$ interval (i.e. the interval from the end of the regional A-wave of the preceding cycle to the beginning of the regional E-wave of the subsequent cycle) at the basal level of each of the above mentioned left ventricular segments $\left(\mathrm{CO}_{\mathrm{IVS}}, \mathrm{CO}_{\mathrm{INF}}, \mathrm{CO}_{\mathrm{POST}}, \mathrm{CO}_{\mathrm{LAT}}, \mathrm{CO}_{\mathrm{ANT}}\right.$, respectively), together with its three systolic components (regional isovolumic contraction time [ICT], regional left ventricular ejection time [LVET], and regional isovolumic relaxation time [IRT]); - the EA interval (i.e. the interval from the beginning of the regional $\mathrm{E}$-wave and the end of the regional A-wave in the same cycle); the Q-S interval (i.e. the interval from the Q-wave to the beginning of regional sustained left ventricular ejection) for each segment; and - the Q-S ${ }_{2}$ interval (i.e. the interval from the Q-wave to the regional sustained left ventricular ejection peak) for each segment. All standard and TDI echocardiographic measurements were made on three cardiac cycles and values were afterwards averaged.

Each of the left ventricular segments was also evaluated qualitatively, describing the contraction pattern as: - I (synchronized), - IIA (unsynchronized of lesser de-

Table 1. Inclusion criteria.

Dilated cardiomyopathy of ischemic or idiopathic
origin

Left bundle branch block or intraventricular conduction delay with the QRS complex prolongation $\geq 150 \mathrm{~ms}$

PQ interval $\geq 160 \mathrm{~ms}$

Left ventricular ejection fraction $\leq 35 \%$

NYHA functional class III or IV

Optimal medical therapy stable for at least 6 weeks prior to inclusion

Prior myocardial infarction older than 8 weeks

Signed written informed consent

Optimal medical therapy included loop diuretics, spironolactone, ACE - inhibitors or AT receptor ${ }_{1}$ blockers and $\beta$ - blockers if tolerated. 
Table 2. Clinical and demographical data of the study population.

\begin{tabular}{|c|c|}
\hline Study population (n) & 50 \\
\hline Age (years) & $69.7 \pm 8.3(49-84)$ \\
\hline Men & $38(76 \%)$ \\
\hline Women & $12(24 \%)$ \\
\hline Ischemic heart disease & $29(58 \%)$ \\
\hline $\begin{array}{l}\text { Idiopathic dilated } \\
\text { cardiomyopathy }\end{array}$ & $21(42 \%)$ \\
\hline Basal QRS width (ms) & $173 \pm 19(150-225)$ \\
\hline Basal PQ interval (ms) & $209 \pm 49(160-340)$ \\
\hline $\begin{array}{l}\text { Minnesota Living with Heart } \\
\text { Failure Score }\end{array}$ & $45 \pm 16$ points \\
\hline NYHA functional class & $3.1 \pm 0.3$ \\
\hline NYHA III & $41(82 \%)$ \\
\hline NYHA IV & $9(18 \%)$ \\
\hline Medication: furosemide & $48(96 \%)$ \\
\hline spironolactone & $39(78 \%)$ \\
\hline hydrochlorothiazide & $5(10 \%)$ \\
\hline$\beta$ - blockers & $45(90 \%)$ \\
\hline ACE - inhibitors & $45(90 \%)$ \\
\hline ATII receptor blockers & $4(8 \%)$ \\
\hline digoxin & $21(42 \%)$ \\
\hline $\begin{array}{l}\text { Left ventricular ejection } \\
\text { fraction }(\%)\end{array}$ & $20.5 \pm 6.5(10-35)$ \\
\hline $\begin{array}{l}\text { Left ventricular end-diastolic } \\
\text { diameter }(\mathrm{mm})\end{array}$ & $68.6 \pm 8.7(50-98)$ \\
\hline $\begin{array}{l}\text { Left ventricular end-diastolic } \\
\text { volume }(\mathrm{ml})\end{array}$ & $210 \pm 79(121-531)$ \\
\hline $\begin{array}{l}\text { Left ventricular end-systolic } \\
\text { volume }(\mathrm{ml})\end{array}$ & $166 \pm 72(83-466)$ \\
\hline $\begin{array}{l}\text { Left atrial dimension } \\
(\text { PLAX view })(\mathrm{mm})\end{array}$ & $46.6 \pm 4.6(37-55)$ \\
\hline
\end{tabular}

gree), - IIB (unsynchronized of greater degree), - IIIA (dyskinetic, reversed early in systole), - IIIB (dyskinetic, reversed late in systole), and - IV (dyskinetic, entirely reversed in the whole systole).

\section{STATISTICAL ANALYSIS}

Statistical analysis was performed using SPSS for MS Windows software. Baseline characteristics were summarized using appropriate descriptive statistics. Analysis of variance (ANOVA) was applied to compare repeated measurements of echocardiographic parameters during baseline, bifocal and biventricular pacing, with Bonferroni correction to adjust the $p$ values. Percentages of hemodynamic improvement during different stimulation modes were compared by the one-way $t$-test and the results were verified by nonparametric Wilcoxon test. Dichotomous variables were compared using the $\chi^{2}$ test or Fisher's exact probability test where appropriate. Linear correlations were calculated by Pearson correlation coefficient. A p value $<0.05$ was considered statistically significant.

\section{RESULTS}

Fifty consecutive patients with indication for cardiac resynchronization therapy (CRT) fulfilling the inclusion criteria (Tab. 1) were included in the study. Underlying disease was idiopathic dilated cardiomyopathy (IDCM) in 21 patients (42\%) and ischemic heart disease (IHD) in 29 patients (58\%). Tab. 2 summarizes other demographic and clinical data of our patient cohort.

\section{Hemodynamic measures}

The LV dp/dt did not change significantly between basal state and RVAP $(p=0.68)$. On the contrary, the LV $\mathrm{dp} / \mathrm{dt}_{\text {max }}$ was increased during BFP compared to the baseline $(13.4 \%$, $95 \%$ CI $9.2-17.6 \%, p<0.0001)$, but more prominent increase was achieved during BVP (29.5\%, $95 \%$ CI 23.7 - $35.4 \%$, p < 0.0001). A similar mean increase of the LV dp/dt max $_{\text {max }}$ during BFP was noted between IDCM and IHD patients (Tab. 3), but there were 7 patients with IHD (24\%), in whom no, or even detrimental, acute hemodynamic effect of BFP was observed, but only 1 such patient (5\%) in the IDCM group (Fig. 2, Fig. 3).

BFP increased the LV dp/dt ${ }_{\max }$ by more than $5 \%$ when compared to the baseline values in 33 patients $(66 \%)$ from the entire group (in 19 patients with IHD and in 14 patients with IDCM, no statistically significant difference between subgroups). Only 23 patients (46\%) in the entire group acutely responded to the BFP by more than a $10 \%$ increase of the $\mathrm{LV} \mathrm{dp} / \mathrm{dt}_{\text {max }}$ (15 patients with IHD and 8 patients with IDCM, no statistically significant difference between subgroups). 3 patients (10\%) with IHD and 4 patients (19\%) with IDCM had more than a $30 \%$ increase of the $\mathrm{LV} \mathrm{dp/dt}$ max during BFP. Strong positive correlation was found between the distance of the right ventricular apical and outflow tract leads and percentage of $\mathrm{dp} / \mathrm{dt}_{\max }$ increase in the IDCM patients $(\mathrm{r}=0.72$, $\mathrm{p}<0.001$ ), but not in the IHD patients (Fig. 2, Fig. 3). The distance between the leads correlated also with the time interval from the sensed local right ventricular apical signal to a signal in the outflow tract $(r=0.498$, $\mathrm{p}<0.001)$.

48 patients (96\%) acutely responded to BVP by more than a $5 \%$ increase of the $\mathrm{LV} \mathrm{dp} / \mathrm{dt}_{\text {max }}$ (27 patients with IHD, 21 patients with IDCM, no statistical difference between groups), and 43 patients ( $86 \%$ ) responded with more than a $10 \%$ increase of the $\mathrm{LV} \mathrm{dp} / \mathrm{dt}_{\max }$ (25 patients with IHD, 18 patients with IDCM, no statistical differ- 


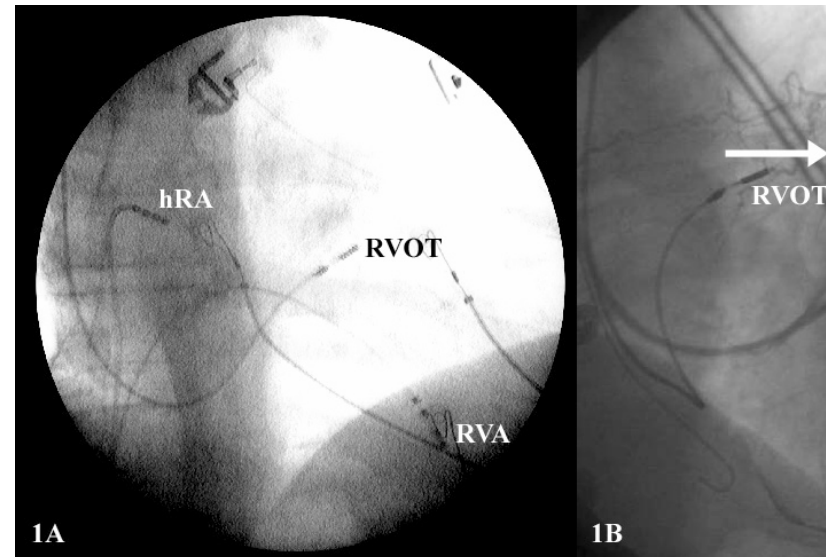

Fig. 1. The example of temporary bifocal stimulation in the anteroposterior projection (Panel 1A) and in the left anterior oblique view $45^{\circ}$ (Panel 1B). Note the orientation of the RVOT lead to the interventricular septum (arrow). hRA...lead in the high right atrium, RVA...lead in the right ventricular apex, RVOT...lead in the right ventricular outflow tract.

ence between groups). In those 7 patients with no acute hemodynamic improvement (delta LV dp/dt ${ }_{\text {max }}$ during BVP less than $10 \%$ ), none, except for 1 patient, positively responded to BFP. In this particular patient with IDCM, the LV dp/dt ${ }_{\text {max }}$ increased from $1152 \mathrm{mmHg} . \mathrm{s}^{-1}$ to $1296 \mathrm{mmHg} . \mathrm{s}^{-1}$ on BFP (by $12.5 \%$ ) and only to 1248 mmHg.s ${ }^{-1}$ on BVP (by $8.3 \%$ ). There were also 8 patients (16\%), in whom the LV dp/dt ${ }_{\text {max }}$ during BFP was equal or greater than $\mathrm{LV} \mathrm{dp} / \mathrm{dt}_{\text {max }}$ during BVP. Interestingly, the majority of such patients ( $75 \%$ ) had IHD.

In the IHD subgroup of patients, there was no difference between patients with previous anterior myocardial infarction and infarction of different localizations in response to bifocal stimulation with respect to acute hemodynamic changes, both when the cut-off value for acute hemodynamic response was defined as a $5 \%$ increase of the $\mathrm{LV} \mathrm{dp} / \mathrm{dt}_{\max }(58 \%$ vs. $70 \%, \mathrm{p}=\mathrm{NS}$ ) and when the value was defined as $10 \%$ ( $58 \%$ vs. $47 \%, \mathrm{p}=\mathrm{NS}$ ).

Aortic pulse pressure was not significantly changed during BFP when compared to the baseline SR values $(0.9 \%, 95 \%$ CI $-2.2-4.0 \%)$, but increased significantly during BVP (7.7 \%, $95 \%$ CI 4.5 - $10.8 \%$, p < 0.001, Tab. 3).

\section{Reproducibility of hemodynamic measures}

Reproducibility of the $\mathrm{LV} \mathrm{dp/dt}$ max measurements was acceptable. Variability of the three measurements fluctuated between $0 \%$ to $2.9 \%(0.36 \pm 0.9 \%), 0 \%$ to $3.1 \%$ $(0.73 \pm 1.23 \%), 0 \%$ to $3.7 \%(1.4 \pm 1.54 \%)$ and $0 \%$ to $3.6 \%(0.6 \pm 1.2 \%)$ during basal state, RVAP, BFP and BVP, respectively. Overall variability of the $\mathrm{LV} \mathrm{dp} / \mathrm{dt}_{\max }$ counted $0.8 \pm 1.3 \%$.

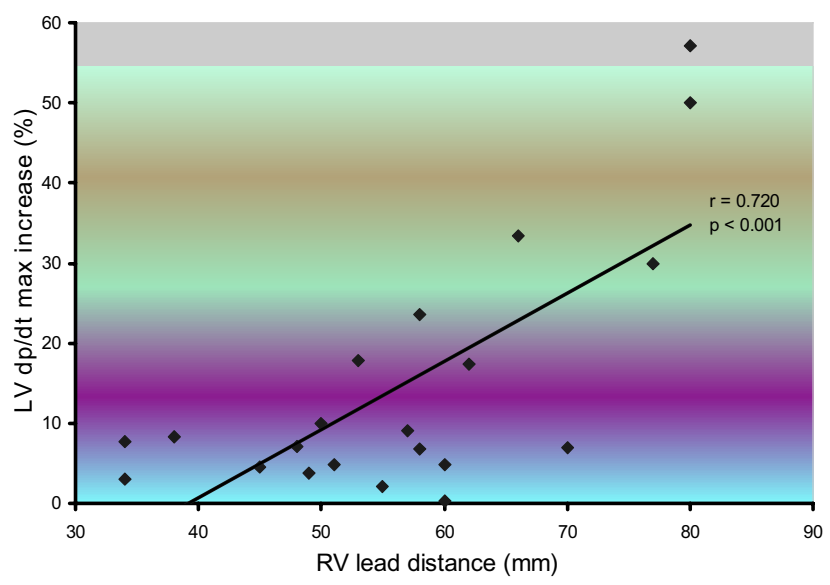

Fig. 2. Relationship between the distance of the right ventricular apical and outflow tract leads and maximum of $\mathrm{dp} / \mathrm{dt}$ in the left ventricle during $\mathrm{AV}$ synchronous bifocal pacing in patients with idiopathic dilated cardiomyopathy.

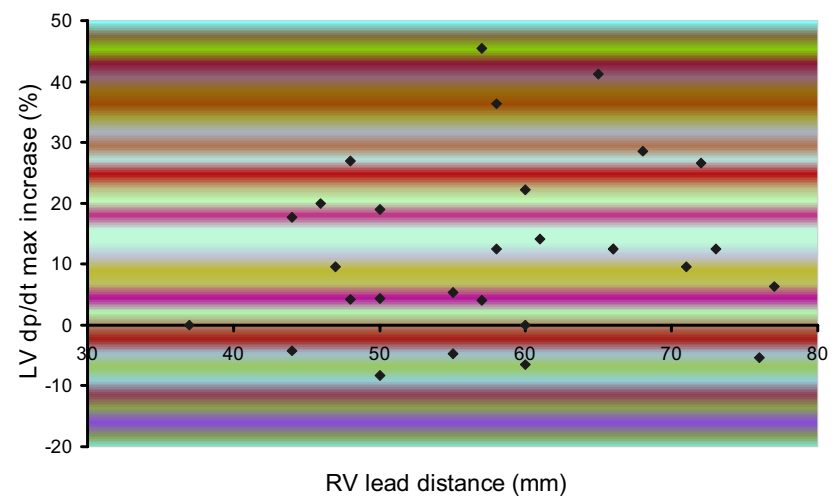

Fig. 3. Relationship between the distance of right ventricular apical and outflow tract leads and the maximum of $\mathrm{dp} / \mathrm{dt}$ in the left ventricle during AV synchronous bifocal pacing in patients with ischemic heart disease.

QRS width and heart rates during hemodynamic measures

Basal QRS width was $173 \pm 19 \mathrm{~ms}$ and both BFP and BVP decreased it significantly $(160 \pm 13 \mathrm{~ms}, \mathrm{p}=0.001$ and $139 \pm 12 \mathrm{~ms}, \mathrm{p}<0.0001$, respectively). Heart rates during various measurements are summarized in Tab. 3. Overall, heart rates varied between different modes of stimulation (basal state, RVAP, BFP, BVP): from 0 to $10 \mathrm{bpm}$, with an average of $5.5 \pm 3.1 \mathrm{bpm}$.

\section{Echocardiographic parameters}

Left ventricular ejection fraction increased significantly during BFP ( $23 \pm 8 \%$ vs. $20 \pm 6 \%$, p < 0.0003 ), but the increase was more prominent during BVP (29 $\pm 8 \%$, $\mathrm{p}<0.0001$ ). Global systolic contraction time ( $\mathrm{CO}$ interval) was significantly shortened both during BFP (445 \pm $52 \mathrm{~ms}, \mathrm{p}=0.001)$ and BVP $(431 \pm 41 \mathrm{~ms}, \mathrm{p}<0.0001)$ in comparison with the $\mathrm{CO}$ interval during intrinsic rhythm 


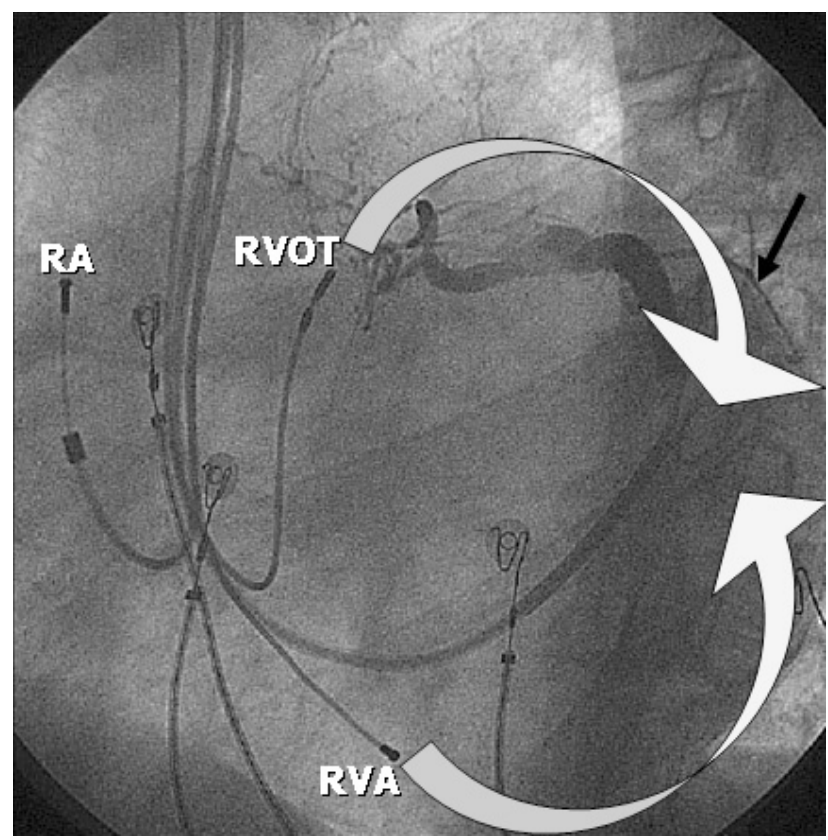

Fig. 4. A case of permanent bifocal pacing. The left ventricular lead could not be inserted into the lateral branch of the coronary sinus due to its very small diameter (black arrow). Spreading of the wavefronts of activation (white arrows) from the right ventricular outflow tract (RVOT) and right ventricular apex (RVA) can significantly preexcite anterior and inferior segments of the left ventricle, thus shortening the total left ventricular activation time.

(476 $\pm 61 \mathrm{~ms}$ ), thus prolonging the LV diastolic filling time (Tab. 4). Myocardial performance index was also significantly decreased in both BFP and BVP $(\mathrm{p}<0.001$ for both).

Interventricular mechanical delay was decreased on BFP compared to the baseline values (43 $\pm 22 \mathrm{~ms}$ vs. $53 \pm 31 \mathrm{~ms}, \mathrm{p}=0.006)$, but BVP produced shorter interventricular delay $(22 \pm 19 \mathrm{~ms}, \mathrm{p}<0.0001$, Tab. 4). In all patients, the regional systolic contraction times (CO intervals in the basal septum, lateral, inferior, posterior and anterior wall) were significantly shortened, corresponding with the prolongation of the respective regional diastolic filling times during both BFP ( $p<0.05$ for all segments) and BVP ( $p<0.001$ for all segments), while the effect of BVP biventricular pacing on regional systole shortening was more pronounced (Tab. 5). The regional myocardial performance index (Tei index) decreased only during the BVP modality ( $\mathrm{p}<0.05$ for all segments, Tab. 6).

In a multivariate analysis of the echocardiographic parameters, none except for the lateral regional $\mathrm{CO}$ interval $\left(\mathrm{CO}_{\mathrm{LAT}}\right)$ at baseline predicted an acute hemodynamic response to bifocal stimulation (when defined as at least a $10 \%$ increase in the $\left.\mathrm{LV} \mathrm{dp} / \mathrm{dt}_{\max }\right)$. Receiver operator curve identified a cut-off value of the $\mathrm{CO}_{\mathrm{LAT}}$ interval less than $530 \mathrm{~ms}$ with $73 \%$ sensitivity, $64 \%$ specificity and $68 \%$ diagnostic accuracy for the response to bifocal stimulation.
Qualitative patterns of the LV segmental contraction significantly improved both during BFP and BVP (Tab. 7). While only $26 \%$ of the LV basal segments showed normal or less desynchronized type of contraction (type I or IIA) at baseline, this number increased to $42 \%$ during BFP and to $79 \%$ during BVP ( $p<0.001)$. Overall, BFP acutely improved qualitative contraction patterns of all basal segments of the left ventricle in $37.6 \%(p<0.0001)$, while this qualitative pattern remained the same in $48.4 \%$ of segments and worsened in $14 \%$ of segments. During BVP, however, the qualitative contraction patterns were acutely improved in $70 \%$ of segments $(p<0.0001)$, while in $24.4 \%$ of segments they remained the same and in $5.6 \%$ of segments they worsened.

Variability of echocardiographic interval measurements was limited below $3 \%$ in the whole study population (below or equal to $10 \mathrm{~ms}$ in absolute values). All but one patient were adequately echocardiographically explorable. In this particular patient, the LV anterior wall could not be properly imaged and the TDI measurements could not be properly done.

\section{DISCUSSION}

Biventricular pacing has recently been proposed for the treatment of patients with advanced heart failure and significant electrical conduction delay between the ventricles and within the left ventricle itself, generally represented by the left bundle branch block. However, various studies report technically unsuccessful procedures in 5$13 \%$ of cases $^{2-4}$. According to the analysis of CONTAK $\mathrm{CHF} / \mathrm{CONTAK}$ CD Biventricular Pacing study ${ }^{4}$, the most frequent causes of coronary venous lead implantation failure were inability to cannulate the coronary sinus (6\%), inability to obtain a stable pacing site ( $5 \%)$, inability to obtain adequate pacing threshold ( $1 \%)$, coronary sinus dissection/perforation ( $1 \%$ ), diaphragmatic stimulation that could not be corrected $(0.2 \%)$ and vascular trauma during attempt at venous access $(0.2 \%)$.

The concept of bifocal pacing as AV synchronous simultaneous right ventricular apical and outflow tract pacing appeared to overcome these pitfalls. The procedure is technically simple, quick and safe and resembles that of standard pacemaker implantation. In addition, it does not require special skills in vascular intervention and/or electrophysiology unlike implantation of the LV lead to the CS tributary. The position of the RVOT lead can easily be fluoroscopically controlled and when using screw-in leads it was proven to be stable and with excellent pacing and sensing parameters during long-term follow-up ${ }^{11,12}$. This potential advantage makes bifocal pacing plausible in patients with LV implantation failure who refuse surgical LV lead placement or in whom such a procedure appears to be too risky.

There are several studies on bifocal pacing published to date, but with conflicting results. Buckingham et al. studied the effect of pacing at the RVOT and the RVA on systolic and diastolic function ${ }^{13}$. In 14 patients with a mean LVEF $32 \pm 4 \%$ the authors failed to show any sig- 
Table 3. Hemodynamic parameters during sinus rhythm, bifocal and biventricular pacing.

\begin{tabular}{|c|c|c|c|c|c|c|c|c|c|}
\hline & \multicolumn{3}{|c|}{ whole group } & \multicolumn{3}{|c|}{ IHD } & \multicolumn{3}{|c|}{ IDCM } \\
\hline & IR & BFP & BVP & IR & BFP & BVP & IR & BFP & BVP \\
\hline heart rate $[\mathrm{bpm}]$ & & & & & & & & & \\
\hline mean & 74 & 75 & 74 & 74 & 74 & 74 & 74 & 75 & 74 \\
\hline SD & 7 & 7 & 6 & 7 & 7 & 6 & 7 & 7 & 6 \\
\hline $\mathbf{d p} / \mathbf{d t}_{\max }\left[\mathrm{mmHg} \cdot \mathrm{s}^{-1}\right]$ & & & & & & & & & \\
\hline mean & 1050 & 1152 & 1296 & 1043 & 1163 & 1329 & 1061 & 1196 & 1345 \\
\hline SD & 268 & 277 & 300 & 248 & 269 & 293 & 299 & 294 & 317 \\
\hline $\mathbf{d p} / \mathbf{d t}_{\max }[\%$ of increase $]$ & & $13.4^{*}$ & $29.5^{\text {क }}$ & & $12.5^{*}$ & $29.4^{\ddagger}$ & & $14.7^{*}$ & $29^{\text {\$ }}$ \\
\hline $95 \% \mathrm{CI}$ & & $9.2-17.6$ & $23.7-35.4$ & & $7.1-17.9$ & $22.1-36.6$ & & $7.5-21.8$ & $19.2-40.3$ \\
\hline $\begin{array}{l}\text { range of } \mathbf{d p} / \mathbf{d t}_{\max } \\
\text { [\% of increase] }\end{array}$ & & $-8-57$ & $2-107$ & & $-8-45$ & $3-82$ & & $0-57$ & $2-107$ \\
\hline Ao-PP $[\mathrm{mmHg}]$ & & & & & & & & & \\
\hline mean & 54 & 54 & 59 & 55 & 55 & 59 & 52 & 53 & 57 \\
\hline SD & 15 & 17 & 17 & 15 & 17 & 17 & 16 & 17 & 17 \\
\hline Ao-PP [\% of increase] & & 0.9 & $7.7^{9}$ & & 0.4 & $6.7^{\#}$ & & 0.5 & 9.0 ฯ \\
\hline $95 \% \mathrm{CI}$ & & $-2.2-4.0$ & $4.5-10.8$ & & $-4.1-4.8$ & $2.0-11.4$ & & $-3.0-6.1$ & $4.6-13.3$ \\
\hline
\end{tabular}

IHD...ischemic heart disease, IDCM...idiopathic dilated cardiomyopathy, IR...intrinsic rhythm, BFP...bifocal pacing, BVP...biventricular pacing, $\mathrm{dp} / \mathrm{dt}_{\max } \ldots$ maximum of the first derivative of the left ventricular pressure over time, Ao-PP....aortic pulse pressure, CI...confidence interval.

${ }^{*} \mathrm{p}<0.0001$ for $\mathrm{dp} / \mathrm{dt}_{\text {max }}$ during bifocal pacing when compared to intrinsic rhythm, ${ }^{*} \mathrm{p}<0.0001 \mathrm{for} \mathrm{dp} / \mathrm{dt}_{\text {max }}$ during biventricular pacing when compared to intrinsic rhythm, ${ }^{\mathrm{p}} \mathrm{p}<0.001$ for aortic pulse pressure during biventricular pacing when compared to intrinsic rhythm. ${ }^{\#} \mathrm{p}=0.007$ for aortic pulse pressure during biventricular pacing when compared to intrinsic rhythm.

Table 4. Echocardiographic parameters during sinus rhythm, bifocal and biventricular AV synchronous pacing.

\begin{tabular}{|l|c|c|c|c|c|c|}
\hline & $\begin{array}{c}\mathrm{EF} \\
(\%)\end{array}$ & $\begin{array}{c}\mathrm{RPEP} \\
(\mathrm{ms})\end{array}$ & $\begin{array}{c}\text { LPEP } \\
(\mathrm{ms})\end{array}$ & $\begin{array}{c}\text { IVMD } \\
(\mathrm{ms})\end{array}$ & $\begin{array}{c}\mathrm{CO}_{\text {GLOB }} \\
(\mathrm{ms})\end{array}$ & $\begin{array}{c}\mathrm{MPI}_{\text {GLOB }} \\
(\mathrm{ms})\end{array}$ \\
\hline IR & $20 \pm 6$ & $117 \pm 28$ & $169 \pm 37$ & $53 \pm 31$ & $476 \pm 61$ & $0.849 \pm 0.244$ \\
\hline $\mathrm{BFP}$ & $23 \pm 8$ & $121 \pm 21$ & $164 \pm 24$ & $43 \pm 22$ & $445 \pm 52$ & $0.689 \pm 0.308$ \\
\hline BVP & $29 \pm 8$ & $124 \pm 28$ & $139 \pm 27$ & $22 \pm 19$ & $431 \pm 41$ & $0.662 \pm 0.238$ \\
\hline $\begin{array}{l}\text { p value } \\
\text { BFP vs. IR } \\
\text { BVP vs. IR }\end{array}$ & \begin{tabular}{c}
0.0003 \\
\hline
\end{tabular} & $\mathrm{NS}$ & $\mathrm{NS}$ & 0.006 & 0.001 & 0.0001 \\
\hline
\end{tabular}

EF...left ventricular ejection fraction, RPEP...right ventricular preejection time, LPEP...left ventricular preejection time, IVMD...interventricular mechanical delay, $\mathrm{CO}_{\mathrm{GLOB}} \ldots$. time interval of the global left ventricular systole, $\mathrm{MPI}_{\mathrm{GLOB}} \ldots . . \mathrm{global}$ myocardial performance index, IR...intrinsic rhythm, BFP...bifocal pacing, BVP...biventricular pacing. 
Table 5. Regional systolic contraction times during sinus rhythm, bifocal and biventricular AV synchronous pacing.

\begin{tabular}{|l|c|c|c|c|c|c|}
\hline & $\begin{array}{c}\mathrm{CO}_{\text {SEP }} \\
(\mathrm{ms})\end{array}$ & $\begin{array}{c}\mathrm{CO}_{\mathrm{LAT}} \\
(\mathrm{ms})\end{array}$ & $\begin{array}{c}\mathrm{CO}_{\text {INF }} \\
(\mathrm{ms})\end{array}$ & $\begin{array}{c}\mathrm{CO}_{\text {POST }} \\
(\mathrm{ms})\end{array}$ & $\begin{array}{c}\mathrm{CO}_{\text {ANT }} \\
(\mathrm{ms})\end{array}$ & $\begin{array}{c}\mathrm{CO}_{\text {PK }} \\
(\mathrm{ms})\end{array}$ \\
\hline IR & $541 \pm 50$ & $526 \pm 66$ & $560 \pm 50$ & $548 \pm 35$ & $525 \pm 56$ & $487 \pm 65$ \\
\hline BFP & $518 \pm 39$ & $513 \pm 60$ & $528 \pm 40$ & $518 \pm 40$ & $500 \pm 45$ & $458 \pm 39$ \\
\hline BVP & $478 \pm 43$ & $492 \pm 65$ & $494 \pm 52$ & $491 \pm 49$ & $466 \pm 49$ & $450 \pm 38$ \\
\hline $\begin{array}{l}\text { p value } \\
\text { BFP vs. IR } \\
\text { BVP vs. IR }\end{array}$ & $<0.01$ & 0.046 & 0.0006 & 0.0008 & 0.011 & 0.004 \\
\hline
\end{tabular}

$\mathrm{CO}_{\mathrm{SEP}} \ldots$ systolic time interval of the basal septal left ventricular segment, $\mathrm{CO}_{\mathrm{LAT}} \ldots$. systolic time interval of the basal lateral left ventricular segment, $\mathrm{CO}_{\mathrm{INF}} \cdots$ systolic time interval of the basal inferior left ventricular segment, $\mathrm{CO}_{\text {POsT }} \cdots$ systolic time interval of the basal posterior left ventricular segment, $\mathrm{CO}_{\mathrm{ANT}} \ldots$...systolic time interval of the basal anterior left ventricular segment, $\mathrm{CO}_{\mathrm{PK}} \ldots$...systolic time interval of the free basal right ventricular segment, IR...intrinsic rhythm, BFP...bifocal pacing, BVP...biventricular pacing.

Table 6. Regional myocardial performance indexes during sinus rhythm, bifocal and biventricular AV synchronous pacing.

\begin{tabular}{|l|c|c|c|c|c|c|}
\hline & MPI $_{\text {SEP }}$ & MPI $_{\text {LAT }}$ & MPI $_{\text {INF }}$ & MPI $_{\text {POST }}$ & MPI $_{\text {ANT }}$ & MPI $_{\text {PK }}$ \\
\hline IR & $1.283 \pm 0.527$ & $1.200 \pm 0.688$ & $1.240 \pm 0.532$ & $1.215 \pm 0.431$ & $1.058 \pm 0.334$ & $0.872 \pm 0.245$ \\
\hline BFP & $1.188 \pm 0.400$ & $1.156 \pm 0.520$ & $1.184 \pm 0.468$ & $1.198 \pm 0.448$ & $1.147 \pm 0.469$ & $0.770 \pm 0.224$ \\
\hline BVP & $0.902 \pm 0.264$ & $1.045 \pm 0.389$ & $0.992 \pm 0.334$ & $0.898 \pm 0.234$ & $0.831 \pm 0.245$ & $0.827 \pm 0.225$ \\
\hline p value & & & & & & \\
BFP vs. IR & $\mathrm{NS}$ & $\mathrm{NS}$ & $\mathrm{NS}$ & $\mathrm{NS}$ & $\mathrm{NS}$ & 0.002 \\
BVP vs. IR & $<0.0001$ & 0.05 & 0.007 & 0.009 & 0.001 & $\mathrm{NS}$ \\
\hline
\end{tabular}

MPI...myocardial performance index, IR...intrinsic rhythm, BFP...bifocal pacing, BVP...biventricular pacing.

Table 7. Qualitative left ventricular contraction patterns at baseline and during bifocal and biventricular AV synchronous pacing.

\begin{tabular}{|c|c|c|c|}
\hline Contraction pattern & Baseline & Bifocal pacing & Biventricular pacing \\
\hline I & 8 & 12 & 116 \\
\hline IIA & 58 & 94 & 47 \\
\hline IIB & 115 & 101 & 2 \\
\hline IIIA & 15 & 12 & 2 \\
\hline IIIB & 38 & 3 & 28 \\
\hline IV & 16 & & 2 \\
\hline
\end{tabular}

Contraction pattern I...synchronized, IIA...unsynchronized of lesser degree, IIB... unsynchronized of greater degree, IIIA...dyskinetic, reversed early in systole, IIIB... dyskinetic, reversed late in systole, IV...dyskinetic, entirely reversed in the whole systole. 
nificant change in the LVEF, cardiac output and peak LV $\mathrm{dp} / \mathrm{dt}$ during intrinsic rhythm, atrial pacing and DVI pacing at the RVA, the RVOT, and both RV sites. However, the highest absolute values of dp/dt were observed during sinus rhythm and the lowest with RVA pacing. This parameter tended to improve progressively with pacing in the RVOT and at both sites (i.e. bifocal pacing). Assuming from the mean QRS width in this study $(107 \pm 32 \mathrm{~ms}$, only 6 of 14 patients had left bundle branch block), the patients had no significant inter- or intraventricular conduction delay. In our study, we dealt with a population of patients traditionally indicated for cardiac resynchronization therapy due to delayed LV activation. Therefore, the results are not directly comparable with ours.

Pachon et a $1{ }^{14}$ studied 39 patients with cardiac failure (NYHA $3.1 \pm 0.8$ ) and pacemaker indication due to chronic AV block, most of them with dilated cardiomyopathy (idiopathic or Chagas disease). After clinical stabilization the patients were studied under three stimulation modes in the same session: RVOT pacing, RVA pacing and bifocal stimulation. In comparison to conventional RVA pacing, BFP increased ejection fraction (12.4\%), cardiac output (19.5\%), and peak filling rate (31.0\%), and decreased QRS duration (24.7\%), left atrium area (11.9\%) and mitral regurgitation area (32.3\%). RVOT stimulation alone showed less obvious results. Moreover, the quality-of-life showed an impressive score reduction of $50.4 \%$.

\section{Hemodynamic benefit}

In our study, the prominent acute hemodynamic response on BFP (when defined as more than $10 \%$ increase

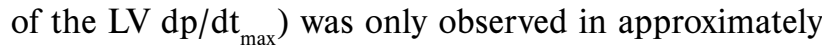
one half of patients both with IHD and with IDCM. The effect of BFP was comparable in both IDCM group and IHD patients. Though it might have been the error of small numbers, there were more patients with zero or even detrimental effects of BFP among the IHD patients than in the IDCM patients. In addition, in both groups of patients there were some patients with excellent immediate improvement in LV contractility and therefore IHD patients should not be automatically considered being more likely non-responders to BFP. It seems reasonable that bifocal pacing should be rather tested in IHD patients for immediate effects prior to its permanent implantation.

The positive influence of both BFP and BVP could not have been caused by the AV delay optimization, since

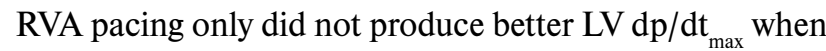
compared to the basal state.

Interestingly, in patients with IDCM, the distance between the right ventricular leads in the RVA and RVOT correlated strongly with the improvement of LV contractility suggesting that the two wavefronts of activation of the left ventricle arising from both right ventricular leads should start as far from each other as possible to achieve the shortest total LV activation time and improve hemodynamics. Lack of such correlation in IHD patients might be explained by less predictable impulse spreading in the left ventricle due to myocardium scarring after infarction.
The best acute hemodynamic result of bifocal pacing was noted in patients, whose duration of regional LV lateral systolic interval was shorter. $\mathrm{CO}_{\mathrm{LAT}}$ longer than $530 \mathrm{~ms}$ predicted worse results of bifocal pacing with respect to acute hemodynamic improvement. It is conceivable that bifocal pacing can shorten total LV activation time predominately in cases where lateral segments are not too delayed and anterior and posterior segments of the left ventricle can be significantly preexcited by wavefronts of activation spreading from the RVOT and RVA leads (Fig. 4).

\section{Cardiac resynchronization benefit}

Both bifocal and biventricular pacing produced significantly improved LVEF, but the change in absolute values was mild on bifocal pacing, while biventricular produced more prominent acute improvement. Interventricular mechanical delay was also significantly improved on bifocal pacing, again with much more improvement during biventricular pacing.

As far as the quantitative TDI analysis of systolic time intervals is concerned, we found a reduction in the duration of the regional systole, and a corresponding prolongation in the duration of the regional diastole at all basal segments of the left ventricle, during bifocal pacing compared to intrinsic rhythm. A clear improvement towards better left ventricular synchrony, as evaluated by qualitative TDI analysis, was also found during bifocal pacing. The effect of biventricular pacing was more pronounced and better when compared with bifocal pacing, nevertheless, such a result supports the evidence that multisite pacing in the right ventricle may also represent a form of effective cardiac resynchronization therapy in cases when placement of the LV lead is not technically feasible. Due to the rationale of cardiac resynchronization therapy, the more the duration of the systole shortens regionally, the more the activation of each of the left ventricular segments becomes synchronous with the others. Thus, better left ventricular synchrony at all segments together with the shortening in the duration of the regional systole contributed to the improvement of the left ventricular efficiency during both bifocal and biventricular pacing. Yet the results of our study support the finding that biventricular pacing generally prevails over bifocal pacing.

\section{Long-term effect of bifocal pacing}

In our study we examined only acute hemodynamic changes during bifocal pacing which cannot automatically be translated into long-term benefits in terms of clinical improvement and/or left ventricular reverse remodeling. However, there are reports in the literature, that bifocal pacing can increase quality of life ${ }^{14}$. The report on the long-term effect of bifocal pacing in patients with dilated cardiomyopathy caused by the Chagas disease is disappointing. Long-term retention of the positive effect of bifocal pacing in weeks after implantation could not be maintained over 6 months follow-up, most likely due to occurrence of malignant ventricular arrhythmias and natural progression of the disease itself ${ }^{15}$. In patients with 
ischemic or idiopathic dilated cardiomyopathy, the longterm effect of bifocal pacing has not yet been thoroughly investigated. O'Donnell followed patients with conventional indications for $\mathrm{CRT}^{16}$. In cases of unsuccessful LV lead implantation the authors used bifocal pacing. All patients were followed for 12 months and the two groups were compared. The authors concluded that both patients with bifocal pacing and patients with biventricular pacing experienced a similar improvement in functional capacity, increase in 6 minutes walking distance, and decreased need for hospitalization. Moreover, though the tissue Doppler indices of LV synchrony improved earlier in the biventricular group, the improvement was similar in both groups at 6 months. The major drawback of this study is that there were only 6 patients in the bifocal group, thus the number of patients for comparison with biventricular pacing group counting 44 patients was limited.

Another major clinical trial in a substudy of 50 patients with bifocal (RVA + RVOT) pacing found no improvement in quality of life, functional class, exercise capacity, and ventricular function after 3 months of pacing. Nonetheless, patients in this ROVA trial with bifocal pacing were not paced from the RVOT and the RVA simultaneously but with a delay of $31 \mathrm{~ms}$, which might have had a significant effect on the outcome. It can be concluded that to date, we lack a prospective double blind cross-over study evaluating the effects of bifocal pacing on clinical status, cardiac hemodynamics and left ventricular reverse remodeling.

\section{LIMITATIONS}

The major limitation of our study was that we did not use tip-manometer catheters during our hemodynamic measurements. However, we meticulously monitored patency of the 6 Fr pigtail catheters that were used for these measurements and were flushed with heparin to prevent any blood clotting and dumping of the signal. We also expressed the $\mathrm{dp} / \mathrm{dt}_{\max }$ in percentage of increase avoiding direct comparison of absolute values.

\section{CONCLUSIONS}

Biventricular pacing is an established form of cardiac resynchronization therapy for patients with depressed LV function, advanced heart failure symptoms and exhausted pharmacological therapy. Nevertheless, the procedure of LV lead implantation is technically demanding and unsuccessful in 5-10\% of cases. It is conceivable that with emerging novel guiding instruments and novel over-the-wire leads together with increasing overall experience with the implantation procedures, the number of LV lead implantation failures will decline, though it will never achieve $0 \%$.

Biventricular stimulation is superior to bifocal with respect to cardiac resynchronization parameters (inter- and/ or intraventricular conduction delay) and acute improve- ment in left ventricular contractility. In some patients, however, it can offer considerable hemodynamic benefit regardless of the etiology of cardiac failure. A positive impact of bifocal pacing can generally be expected in patients with idiopathic dilated cardiomyopathy while in patients with ischemic heart disease it should be verified before implantation of permanent bifocal pacing, since approximately one quarter of such patients will experience no, or even detrimental, effects of this pacing modality.

Typically, candidates for bifocal pacing will be recruited from a cohort of patients, in which the implantation of the LV lead is not technically feasible and who refuse to undergo surgical epicardial placement of the LV lead and/or are at a high risk for such procedures performed under general anesthesia. Perspective candidates should not have the duration of the regional systolic interval of the LV lateral wall exceeding 530 ms. Electrodes in the right ventricle should be placed at the longest achievable distance. Further studies are needed to evaluate long-term benefits of this simplified form of cardiac resynchronization therapy with regards to improved clinical status, morbidity, echocardiographic parameters of cardiac function and left ventricular reverse remodeling.

\section{REFERENCES}

1. Abraham WT. Cardiac resynchronization therapy: a review of clinical trials and criteria for identifying the appropriate patient. Rev Cardiovasc Med 2003; 4:S30-S37.

2. Bulava A, Lukl J. Resynchronization therapy in the treatment of congestive heart disease - a review of literature. Int Med Prax 2005; 7:229-236

3. Gras D, Leclercq C, Tang AS, Bucknall C, Luttikhuis HO, KirsteinPedersen A. Cardiac resynchronization therapy in advanced heart failure the multicenter InSync clinical study. Eur J Heart Fail 2002; 4:311-320.

4. Knight BP, Desai A, Coman J, Faddis M, Yong P. Long-term retention of cardiac resynchronization therapy. J Am Coll Cardiol 2004; 44:72-7.

5. Giudici MC. Right ventricular outflow tract pacing improves hemodynamics in patients with class III-IV heart failure and existing apical leads. Pacing Clin Electrophysiol 1998; 21: (Abs) 751.

6. Pachon JC, Pachon EI, Albornoz RN, Pachon JC, Kormann DS, Gimenes VM, Medeiros PT, Silva MA, Sousa JE, Paulista PP, Souza LC, Jatene AD. Ventricular endocardial right bifocal stimulation in the treatment of severe dilated cardiomyopathy heart failure with wide QRS. Pacing Clin Electrophysiol 2001; 24:1369-1376.

7. Henry WL, DeMaria A, Gramiak R, King DL, Kisslo JA, Popp RL, Sahn DJ, Schiller NB, Tajik A, Teichholz LE, Weyman AE. Report of the American Society of Echocardiography Committee on Nomenclature and Standards in Two-dimensional Echocardiography. Circulation 1980; 62:212-217.

8. Quinones MA, Otto CM, Stoddard M, Waggoner A, Zoghbi WA, Doppler Quantification Task Force of the Nomenclature and Standards Committee of the American Society of Echocardiography. Recommendations for quantification of Doppler echocardiography: a report from the Doppler Quantification Task Force of the Nomenclature and Standards Committee of the American Society of Echocardiography. J Am Soc Echocard 2002; 15:167-184.

9. Kim WH, Otsuji Y, Seward JB, Tei C. Estimation of left ventricular function in right ventricular volume and pressure overload. Detection of early left ventricular dysfunction by Tei index. Japanese Heart Journal 1999; 40:145-154.

10. Ansalone G, Giannantoni P, Ricci R, Trambaiolo P, Laurenti A, 
Fedele F, Santini M. Doppler myocardial imaging in patients with heart failure receiving biventricular pacing treatment. Am Heart J 2001; 142:881-896.

11. Res JC, Bokern MJ, Vos DH. Characteristics of bifocal pacing right ventricular apex versus outflow tract. An interim analysis Pacing Clin Electrophysiol 2005; 28:S36-S38.

12. Stambler BS, Ellenbogen K, Zhang X, Porter TR, Xie F, Malik R, Small R, Burke M, Kaplan A, Nair L, Belz M, Fuenzalida C, Gold M, Love C, Sharma A, Silverman R, Sogade F, Van Natta B, Wilkoff BL. Right ventricular outflow versus apical pacing in pacemaker patients with congestive heart failure and atrial fibrillation. J Cardiovasc Electrophysiol 2003; 14:1180-1186.

13. Buckingham TA, Candinas R, Attenhofer C, Van Hoeven H, Hug R, Hess O, Jenni R, Amann FW. Systolic and diastolic function with alternate and combined site pacing in the right ventricle. Pacing Clin Electrophysiol 1998; 21:1077-1084.

14. Pachon JC, Pachon EI, Albornoz RN, Pachon JC, Kormann DS, Gimenes VM, Medeiros PT, Silva MA, Sousa JE, Paulista PP, Souza LC, Jatene AD. Ventricular endocardial right bifocal stimulation in the treatment of severe dilated cardiomyopathy heart failure with wide QRS. Pacing Clin Electrophysiol 2001; 24:1369-1376.

15. da Silva MA. Outcome of right ventricular bifocal pacing in patients with permanent atrial fibrillation and severe dilated cardiomiopathy due to Chagas disease: three years of follow-up. J Interv Card Electrophysiol 2004; 11:193-198.

16. O'Donnell D, Nadurata V, Hamer A, Kertes P, Mohammed W. Bifocal right ventricular cardiac resynchronization therapies in patients with unsuccessful percutaneous lateral left ventricular venous access. Pacing Clin Electrophysiol 2005; 28:S27-S30. 